\title{
Evolutionary Models for Low Mass Stars and Brown Dwarfs at Young Ages
}

\author{
Isabelle Baraffe, Gilles Chabrier, France Allard \\ CRAL, Ecole Normale Supérieure de Lyon, France \\ Peter Hauschildt \\ Center for Simulational Physics, University of Georgia Athens, GA
}

\begin{abstract}
We analyse evolutionary tracks at young ages for low mass stars with masses $m \leq 1.4 \mathrm{M}_{\odot}$ and brown dwarfs down to one mass of Jupiter. We analyse current theoretical uncertainties due to initial conditions. Simple tests on initial conditions show the high uncertainties of models at ages $\lesssim 1 \mathrm{Myr}$.
\end{abstract}

\section{Introduction}

Numerous surveys devoted to the search for substellar objects have been conducted in young clusters with ages spanning from $\sim 1-10 \mathrm{Myr}$, providing a wealth of data for pre-Main Sequence (PMS) objects. The reliability of the current theory for very low mass stars (VLMS) and brown dwarfs (BD) allows now a thorough analysis of such young objects. Unlike to older Main Sequence stars and BDs, comparison between observations and models for very young objects presents some difficulties: (i) extinction due to the surrounding dust modifies both the intrinsic magnitude and the colours of the object, and (ii) the evolution and spectrum of very young objects $(t \lesssim 1 \mathrm{Myr})$ may still be affected by the presence of an accretion disk or circumstellar material residual from the protostellar stage.

This contribution is devoted to models at early ages for VLMS and BDs down to the planetary mass regime. We discuss the uncertainties of the models of Baraffe et al. (1998, BCAH98) and Chabrier et al. (2000, CBAH00) at young ages, and analyse their comparison with observations.

\section{Evolutionary tracks}

The models analyzed in the present paper are based on the input physics already described in BCAH98 and CBAH00. Both sets of models use the same ingredients describing the stellar interior but use different sets of atmosphere models, which provide the outer boundary conditions and the synthetic spectra. The BCAH98 evolutionary tracks are based on the non-grey atmosphere models by Hauschildt, Allard and Baron (1999). These models are dust-free and are appropriate for the description of objects with effective temperatures $T_{\text {eff }} \gtrsim 2300$ 
$\mathrm{K}$. The CBAH00 models are based on atmospheres including the formation and opacity of dust (Allard et al. 2001, hereafter DUSTY models ). As illustrated in CBAH00, dust must be taken into account in order to explain the near-IR colors of late M-dwarfs and L-dwarfs. The latter models are thus more appropriate to the description of objects with $T_{\text {eff }} \lesssim 2300 \mathrm{~K}$. As emphasized in CBAH00, the DUSTY models are not appropriate for the description of spectral and photometric properties of methane dwarfs $\left(T_{\text {eff }}<1600 \mathrm{~K}\right)$, which require a different treatment of dust (the so-called COND models in CBAH00; Allard et al. 2001; Baraffe et al. 2003, in preparation).

The BCAH98 grid covers a mass range from $0.02 \mathrm{M}_{\odot}$ to $1.4 \mathrm{M}_{\odot}$, for ages $\geq 1 \mathrm{Myr}$ up to the Main Sequence for stars. The CBAH00 grid covers masses from $0.001 \mathrm{M}_{\odot}$ to $0.1 \mathrm{M}_{\odot}$ for ages $\geq 1 \mathrm{Myr}$. Figure 1 presents the complete grid of models in a Hertzsprung-Russell diagram (HRD) from $0.001 \mathrm{M}_{\odot}$ to $1.4 \mathrm{M}_{\odot}$.

Objects below $2 M_{\mathrm{J}}$ evolve essentially with $T_{\text {eff }}<1600 \mathrm{~K}$ (see Fig. 1), even at very early ages. Their atmospheric properties are thus better described by the COND models.

As shown in CBAH00, grains bear little effects on the evolution of $L(t)$ and $T_{\text {eff }}(t)$, because of the reduced dependence of evolution upon opacity. We verified that the difference between the different $\mathrm{TiO}$ and $\mathrm{H}_{2} \mathrm{O}$ molecular linelists used in BCAH98 and CBAH00 models (see $\S 3$ in CBAH00), respectively, affect essentially the outer atmospheric layers, and thus the synthetic spectra and colors, but not the deeper atmospheric layers, and thus the outer boundary conditions. The effect of these different molecular linelists on the evolution of the effective temperature $T_{\text {eff }}(t)$ and the bolometric luminosity $L(t)$ is small, less than $100 \mathrm{~K}$ in $T_{\text {eff }}$ and $10 \%$ in $L$ at a given age. As stressed in CBAH00 and Baraffe et al. (2002), the computation of more reliable $\mathrm{H}_{2} \mathrm{O}$ and, to a lesser extent, TiO linelists is badly needed to solve this shortcoming in the present theory.

\section{Uncertainties due to initial conditions}

Although shortcomings still remain in current molecular opacities, the resulting uncertainty on the evolution is small. The treatment of convection remains an important source of uncertainty, above all for masses $m \gtrsim 0.6 \mathrm{M}_{\odot}$ (see Chabrier and Baraffe 2000; Baraffe et al. 2002). One of the main sources of uncertainty for models at early stages of evolution is the choice of the initial conditions. Most of low mass pre-Main Sequence (PMS) models available in the literature (D'Antona \& Mazzitelli 1994, 1997; Burrows et al. 1997; BCAH98; Siess et al. 2000) start from arbitrary initial conditions, totally independent of the outcome of the prior proto-stellar collapse and accretion phases. The initial configuration is that of a fully convective object starting its contraction along the Hayashi line from arbitrary large radii. Evolution starts prior to or at central deuterium ignition, with initial central temperature $\lesssim 10^{6} \mathrm{~K}$. According to studies of low-mass protostellar collapse and accretion phases, such initial conditions are oversimplified, and low mass objects should rather form with relatively small radii (Hartmann et al. 1997, and references therein). Based on spherical accretion protostellar models, Stahler $(1983,1988)$ defined a birthline in the Herztsprung-Russell diagram where young objects become visible. Evolutionary tracks should then start from 


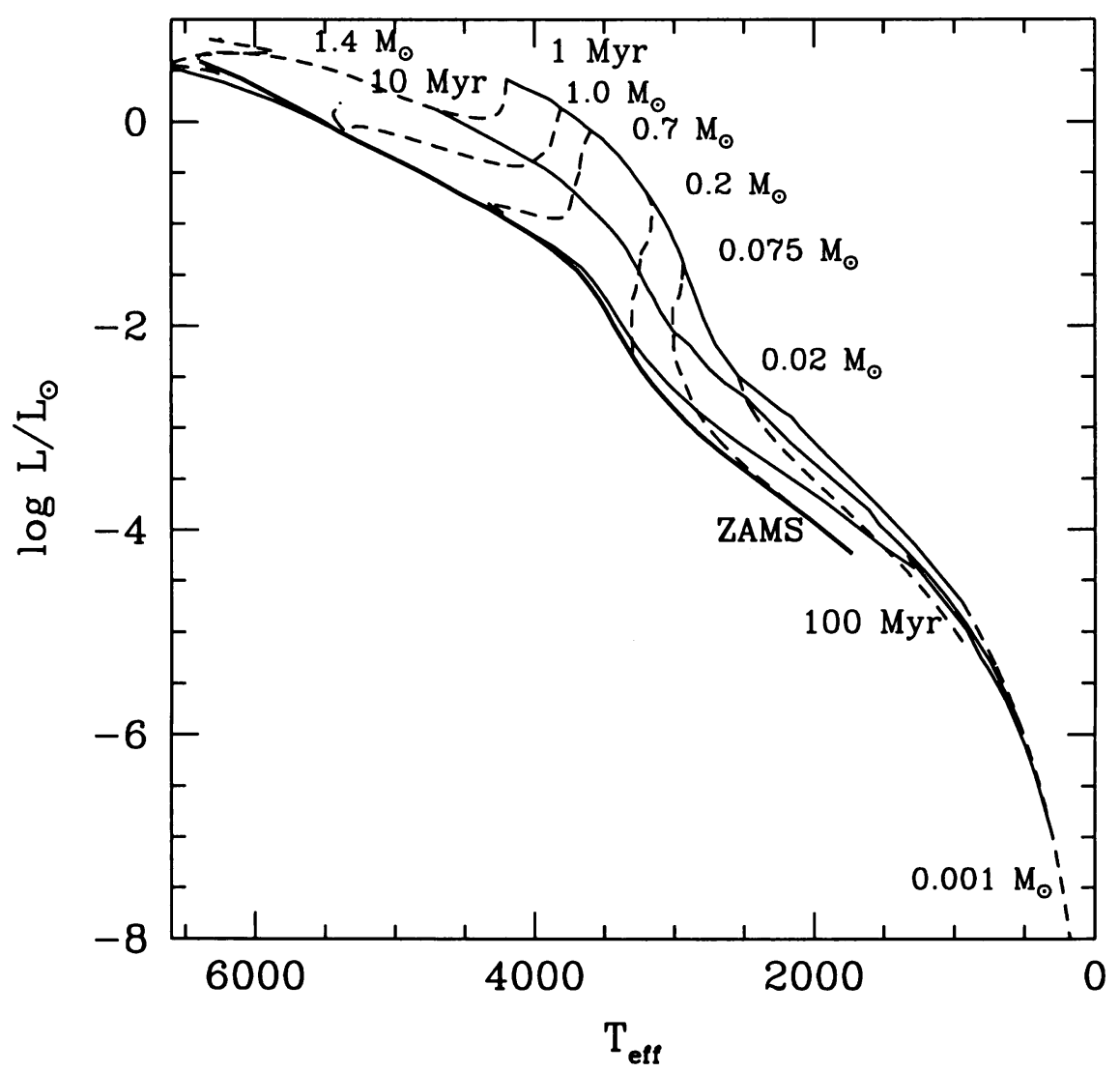

Figure 1. Evolutionary tracks in the Hertzsprung-Russell diagram for masses from $1.4 \mathrm{M}_{\odot}$ to $0.001 \mathrm{M}_{\odot}$ (dashed lines) and ages spanning from $1 \mathrm{Myr}$ to the ZAMS (for stars). Several isochrones for 1, 10 and $100 \mathrm{Myr}$ are indicated by solid lines from right to left. The location of the ZAMS for stars down to $0.075 \mathrm{M}_{\odot}$ is also indicated (left solid line). 
this birthline, which fixes the age $t=0$. Ages determined from models based on the above-mentioned oversimplified conditions should then be corrected accordingly, with substantial corrections for systems younger than a few Myr (see Palla and Stahler 1999). Furthermore, collapse and accretion are unlikely to proceed spherically. Spherical collapse does not consider angular momentum transport, an important issue of the early phases, which affects the subsequent cooling and formation of the protostar. Hartmann et al. (1997) recently illustrate the sensitivity of the birthline locus assuming that accretion proceeds through a disk rather than spherically. This work stresses again the high uncertainty of assigning ages from HRD positions for the youngest objects. Such analysis demonstrates convincingly that assigning an age to objects younger than a few Myr is totally meaningless when the age is based on models using oversimplified initial conditions.

As shown also in Baraffe et al. (2002), for $t \lesssim 1 \mathrm{Myr}$, the evolutionary tracks themselves are sensitive to the initial conditions, whereas after a few Myr, the models converge toward the same track. To illustrate such effect, Baraffe et al. (2002) construct a first set of models with initial radii fixed to obtain initial surface gravities $\log g \sim 3-3.5$ and initial thermal time-scales $t_{t h} \sim$ a few Myr. Such initial conditions are similar to that used in BCAH98 and CBAH00. A second set of models starts with larger radii such that the initial surface gravity $\log g \sim 2.5$. These initial models are more luminous than the previous ones, with central temperatures below $510^{5} \mathrm{~K}$ and initial thermal time-scale $t_{t h} \sim 10^{5}$ yr. We also analyze (see Baraffe et al. 2002 for details) the sensitivity of the models to the mixing length $l_{\text {mix }}$, characteristic of the mixing length formalism (MLT) used to describe convection.

'The effect of different initial radii on evolutionary tracks in a HRD is displayed in Figure 2. Models starting with the lowest gravity are cooler by up to several hundreds $\mathrm{K}$ compared to initially denser, less luminous models with the same mass. Note also that for the second set of models, $T_{\text {eff }}$ increases during the early evolution, under contraction, in contrast to the first set of models with initial $\log g \gtrsim 3.0$. This is the consequence of the different surface gravities, which strongly affect the atmosphere profiles for $T_{\text {eff }}=2200-3500 \mathrm{~K}$. Note that evolution along the Hayashi line does not necessarily proceed at constant $T_{\text {eff }}$ and the common picture of vertical (constant $T_{\text {eff }}$ ) Hayashi tracks is therefore an oversimplified picture of PMS evolution (see Baraffe et al. 2002 for details).

The two sets of models based on different initial radii follow the same track for a given mass and $\alpha_{\text {mix }}$, but do not reach the same position at the same age. Significant differences appear at ages $\lesssim 1$ Myr but vanish after a few Myr. We thus consider $1 \mathrm{Myr}$ as the characteristic time required to forget our arbitrary initial conditions and below which models are too sensitive to input physics and thus too uncertain. This is the main reason why we provide confidently evolutionary models for ages $t \geq 1 \mathrm{Myr}$. To solve this substantial uncertainty requires the consistent evolution between the $3 \mathrm{D}$ collapse of the protostellar phase and the subsequent PMS evolution. 


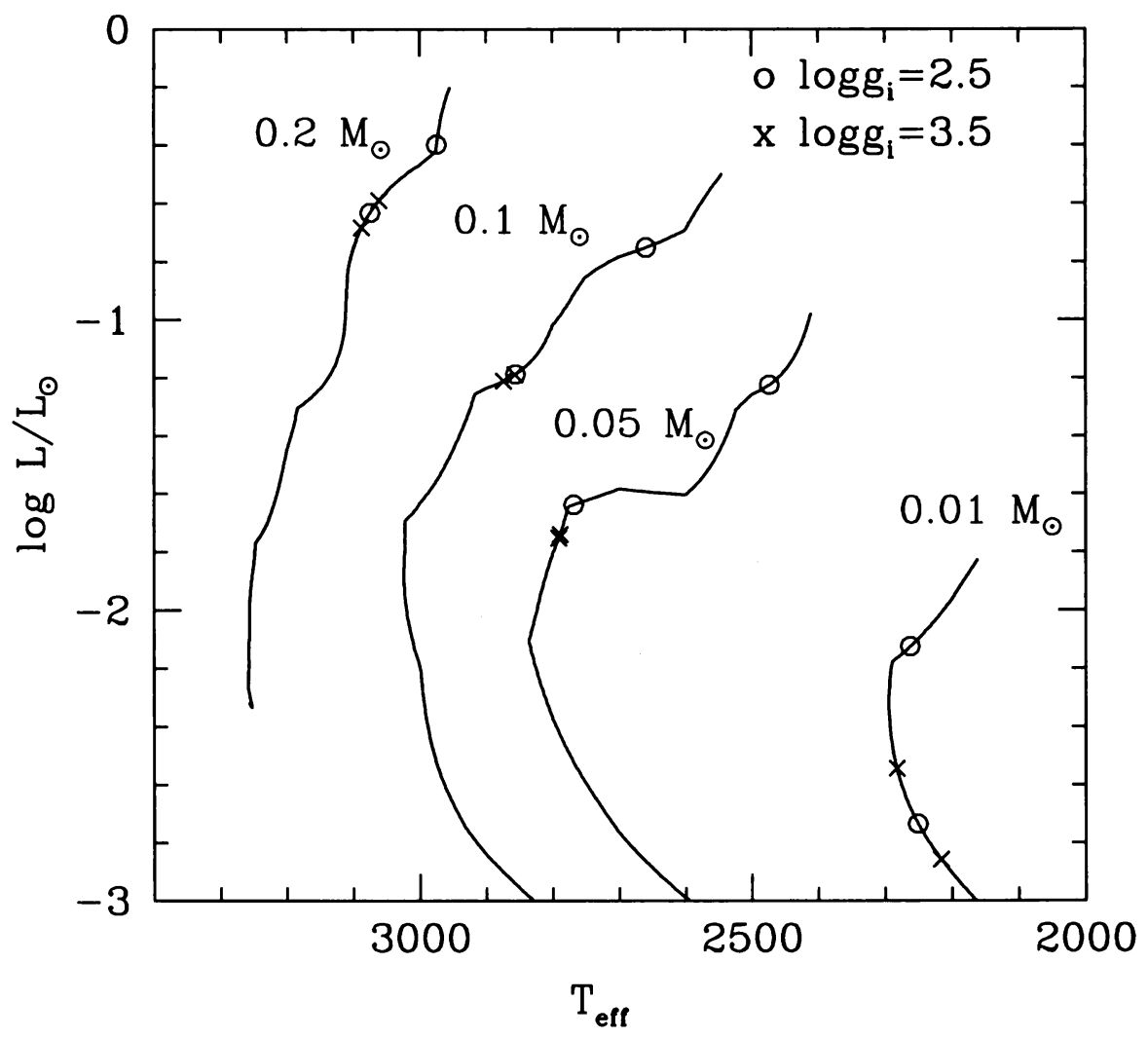

Figure 2. Evolutionary tracks in the Hertzsprung-Russell diagram for masses from $0.2 \mathrm{M}_{\odot}$ to $0.01 \mathrm{M}_{\odot}$, as indicated, and mixing length parameter $\alpha_{\text {mix }}=\left(l_{\text {mix }} / H_{\mathrm{P}}\right)=1$. Ages of $0.1 \mathrm{Myr}$ (upper symbol on the curves) and $1 \mathrm{Myr}$ (lower symbol) are indicated by respectively open circles for models with initial gravity $\log g=2.5$ and crosses for models with initial $\log g=3-3.5$. Note that for the later set of models the initial position $($ age $=0)$ is essentially the same as the position at $0.1 \mathrm{Myr}$ (first cross) and can differ significantly from the position of the former set of models at $t=0$. 


\section{Observational tests}

A better knowledge of initial conditions may come from the determination of the minimum age below which current models start to depart significantly from observations. Estimation of this age can constrain the characteristic time-scales and accretion rates of the protostellar collapse phase. Unfortunately, direct comparisons of observations with models directly in colour - magnitude diagrams are extremely uncertain due to the large extinction in star formation regions, which affects the observed energy distribution and thus the spectra and the colors. Only very few exceptions, such as $\sigma$ Orionis, exhibit low extinction. Recently, Béjar et al. (1999), Zapatero Osorio et al. (1999, 2000) and Martín et al. (2001) obtained optical and near-IR photometry for low mass objects in this cluster. In a $(I-J)$ vs $M_{\mathrm{I}} \mathrm{CMD}$, the data lie between the 1 and $10 \mathrm{Myr}$ isochrones, respectively, for masses down to $\sim 0.01 \mathrm{M}_{\odot}$, using the BCAH98 and CBAH00 models (Zapatero Osorio et al. 2000; Béjar et al. 2001). If statistics is improved and if the membership of the objects to the cluster is confirmed, such observations provide an unique opportunity to test directly the validity of young theoretical isochrones. They also offer the best chance to determine the Mass Function (MF) down to the substellar regime and the minimum mass formed by a collapse process (see Béjar et al. 2001).

Young multiple systems provide also excellent tests for PMS models at young ages, because of the assumed coevality of their different components. In addition, another strong constraint is supplied by the estimate of dynamical masses deduced either from binary systems (Covino et al. 2000; Steffen et al. 2001) or determined from the orbital motion of circumstellar/circumbinary disks (Simon, Dutrey \& Guilloteau 2000). An example is provided by the quadruple system GG TAU (White et al. 1999), with components covering the whole massrange of VLMS and BDs from $1 \mathrm{M}_{\odot}$ to $\sim 0.02 \mathrm{M}_{\odot}$. Orbital velocity measurements of the circumbinary disk surrounding the two most massive components imply a constraint on their combined stellar mass. This mass constraint and the hypothesis of coevality provides a stringent test for PMS models. The BCAH98 models are the most consistent with GG Tau (for details see White et al 1999; Luhman 1999) and provide the closest agreement with derived masses of other young systems (see Figure 3 and Baraffe et al. 2002).

Most of the observed systems displayed in Figure 3 are better reproduced by tracks using a large value of $\alpha_{\text {mix }}(=1.9)$. However, for some systems, such as 1 (Covino et al. 2000), 2 (Steffen et al. 2001) and 4 (BP Tau from Simon et al. 2000), a better agreement is obtained with $\alpha_{\text {mix }}=1$. Although a variation of $\alpha_{\text {mix }}$ with effective temperature and gravity is possible, as suggested by the simulations of Ludwig et al. (1999), none of these three systems occupies a peculiar position in $\left(T_{\text {eff }}, g\right)$ to suggest a different value of $\alpha_{\text {mix }}$. This puzzle may reflect the uncertainties of PMS models based on arbitrary initial conditions. It may also be due to the large uncertainties of observationally-derived spectral type classifications, luminosity estimates and $T_{\text {eff }}$ calibrations for such very young objects. Although still very preliminary, the comparison of observed and synthetic spectra, as recently done by Lucas et al. (2001) for Orion objects, provides a promising way to define a $T_{\text {eff }}$ - scale for very young objects.

Observations of multiple systems, as displayed in Fig.3, are very promising to provide good constraints on PMS tracks and on protostellar collapse models. 
The calculations by Wuchterl \& Klessen (2001) describing the formation and early evolution of a $1 \mathrm{M}_{\odot}$ star yield a track in the HRD which is too hot, by $\sim 700^{\circ}-800 \mathrm{~K}$ in $T_{\text {eff }}$ at a given $L$, compared to observed young systems with masses around a solar mass. Such overestimate of the effective temperature may be due to the assumption of spherical accretion in these calculations.

\section{Conclusion}

The very good agreement of present models based on improved physics with observations for relatively old ( $\mathrm{t} \gtrsim 100 \mathrm{Myr}$ ) low-mass objects yields confidence in the underlying theory. Such evolutionary models can now be confronted to the complex realm of very young objects, providing important information on star formation processes and initial conditions for PMS models. Although based on extremely simple initial conditions (no accretion phase, no account of protostellar collapse phase and time scale, spherical symmetry), these models provide the most accurate comparison with present observations of very young objects (dynamical masses, tests of coevality in multiple systems, CMDs). Given the combining effects of large observational and theoretical uncertainties at very young ages, however, one must remain cautious. It is probably too premature to conclude on the validity of the present models at early phases of evolution.

Realistic initial conditions can only be provided by multi-dimensional protostar collapse simulations, not by spherically-symmetric models. Because of numerical subtleties and complex physical processes (accretion fronts, turbulent time-dependent convection, hydrodynamical radiative transfer, magnetic field etc...), the construction of star formation models is a harsh task, which very likely will necessitate several years of efforts. Besides these theoretical difficulties, observations of very young objects can provide only limited guidance to such simulations, since most phases involved during the collapse are embedded in dusty cocoons. Only the final product can be observationally tested, when the protostar becomes visible. This stage marks essentially the beginning of PMS evolutionary tracks. PMS tracks tested against observations thus provide a precious link to gather insight about star formation models from subsequent evolution.

\section{References}

Allard, F., Hauschildt, P.H., Alexander, D.R., Tamanai, A., Schweitzer, A. 2001, ApJ, 556, 357

Baraffe I., Chabrier G., Allard F., Hauschildt P.H. 1998, A\&A, 337, 403 (BCAH98)

Baraffe I., Chabrier G., Allard F., Hauschildt P.H. 2001, From darkness to light: origin and evolution of young stellar clusters, ASP Conf. Series, Vol. 243, Cargese 2000, p. 571

Baraffe I., Chabrier G., Allard F., Hauschildt P.H. 2002, A\&A, 382, 563

Béjar, V.J.S., Zapatero Osorio M.R., Rebolo R. 1999, ApJ, 521, 671 


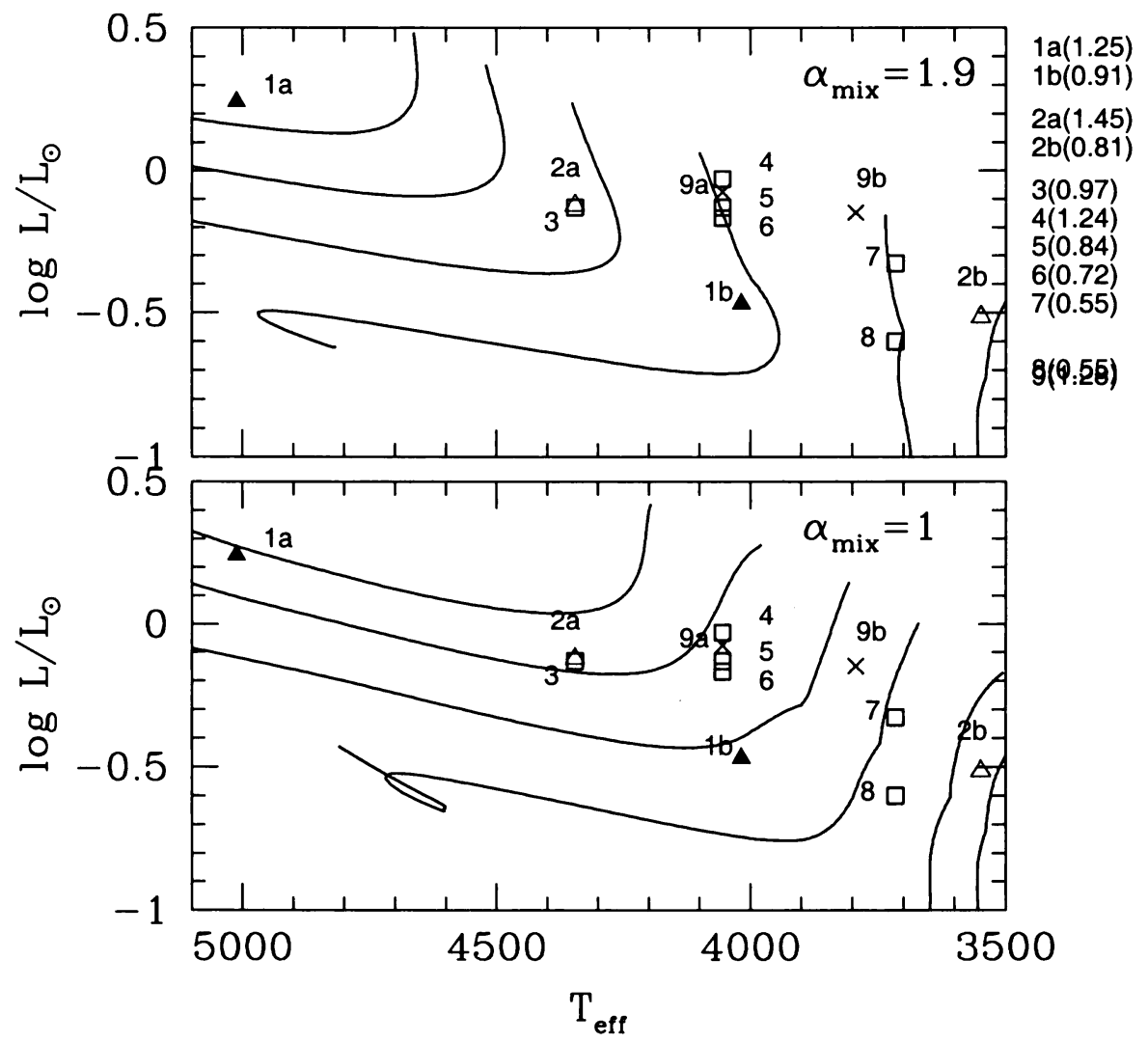

Figure 3. Comparison of evolutionary tracks with observed PMS objects with derived masses. The BCAH98 tracks are displayed for 1.4, $1.2,1,0.8,0.6$ and $0.5 \mathrm{M}_{\odot}$ (from left to right) for two values of $\alpha_{\text {mix }}$. Observations are from Covino et al. (2000, filled triangles), Simon et al. (2000, crosses and open squares) and Steffen et al. (2001, open triangles). Open squares are single objects whereas all the other symbols indicate binaries. For each systems, masses are indicated (in $\mathrm{M}_{\odot}$ ) on the right hand side of the figure. For GG Tau A $(9 a+9 b$, crosses) the total mass is indicated. 
Béjar, V.J.S., Martín, E.L., Zapatero Osorio, M.R., Rebolo, R., Barrado y Navascués, D., Bailer-Jones, C.A.L., Mundt, R., Baraffe, I., Chabrier, G., Allard, F. 2001, ApJ, 556, 830

Burrows A., Liebert, J. 1993, Rev. Mod. Phys., 65, 301

Burrows A., Marley M., Hubbard W.B., Lunine, J.I., Guillot, T., Saumon, D., Freedman, R., Sudarsky, D., Sharp, C. 1997, ApJ, 491, 856 (B97)

Chabrier, G., Baraffe, I. 2000, ARA\&A, 38, 337

Chabrier, G., Baraffe, I., Allard, F., Hauschildt, P.H. 2000, ApJ, 542, 464 (CBAH00)

Covino, E., Catalano, S., Frasca, A., Marilli, E., Fernández, M., Alcala, J.M., Melo, C., Paladino, R., Sterzik, M.F., Stelzer, B. 2000, A\&A, 361, L49

D'Antona, F. and Mazzitelli, I, 1994, ApJS, 90, 467

D'Antona, F. and Mazzitelli, I, 1997, in "Cool stars in Clusters and Associations", ed. R. Pallavicini and G. Micela, Mem. S. A. It., 68, 807

Hartmann, L., Cassen, P., Kenyon, S.J. 1997, ApJ, 475, 770

Hauschildt P.H., Allard F., Baron E. 1999, ApJ, 512, 377

Lucas, P.W., Roche, P.F., Allard, F., Hauschildt, P.H. 2001, MNRAS, 326, 695

Ludwig, H.G., Freytag, B., \& Steffen, M. 1999, A\&A, 346, 111

Luhman KL. 1999, ApJ, 525, 466

Martín, E.L., Zapatero Osorio, M.R., Barrado y Navascués, D., Béjar V.J.S., Rebolo, R., 2001, ApJ, 558, L117

Palla, F., \& Stahler, S.W. 1999, ApJ, 525, 772

Siess, L., Dufour, E., \& Forestini, M. 2000, A\&A, 358, 593

Simon, M., Dutrey, A., \& Guilloteau, S. 2000, ApJ, 545, 1034

Stahler, S.W., 1983, ApJ, 274, 822

Stahler, S.W., 1988, ApJ, 332, 804

Steffen, A.T., Mathieu, R.D., Lattanzi, M.G., Latham, D.W., Mazeh, T., Prato, L., Simon, M., Zinnecker, H., Loreggia, D. 2001, AJ, 122, 997

White R.J., Ghez A.M., Reid I.N., Schultz G. 1999, ApJ, 520, 811

Wuchterl, G., Klessen, R.S. 2001, ApJ, 560, L185

Zapatero Osorio, M.R., Béjar V.J.S., Rebolo, R., Martín, E.L., Basri, G. 1999, ApJ, 524, 115

Zapatero Osorio, M.R., Béjar V.J.S., Martín, E.L., Rebolo, R., Barrado y Navascués, D., Bailer-Jones, C.A.L., Mundt, R. 2000, Science, 290, 103 


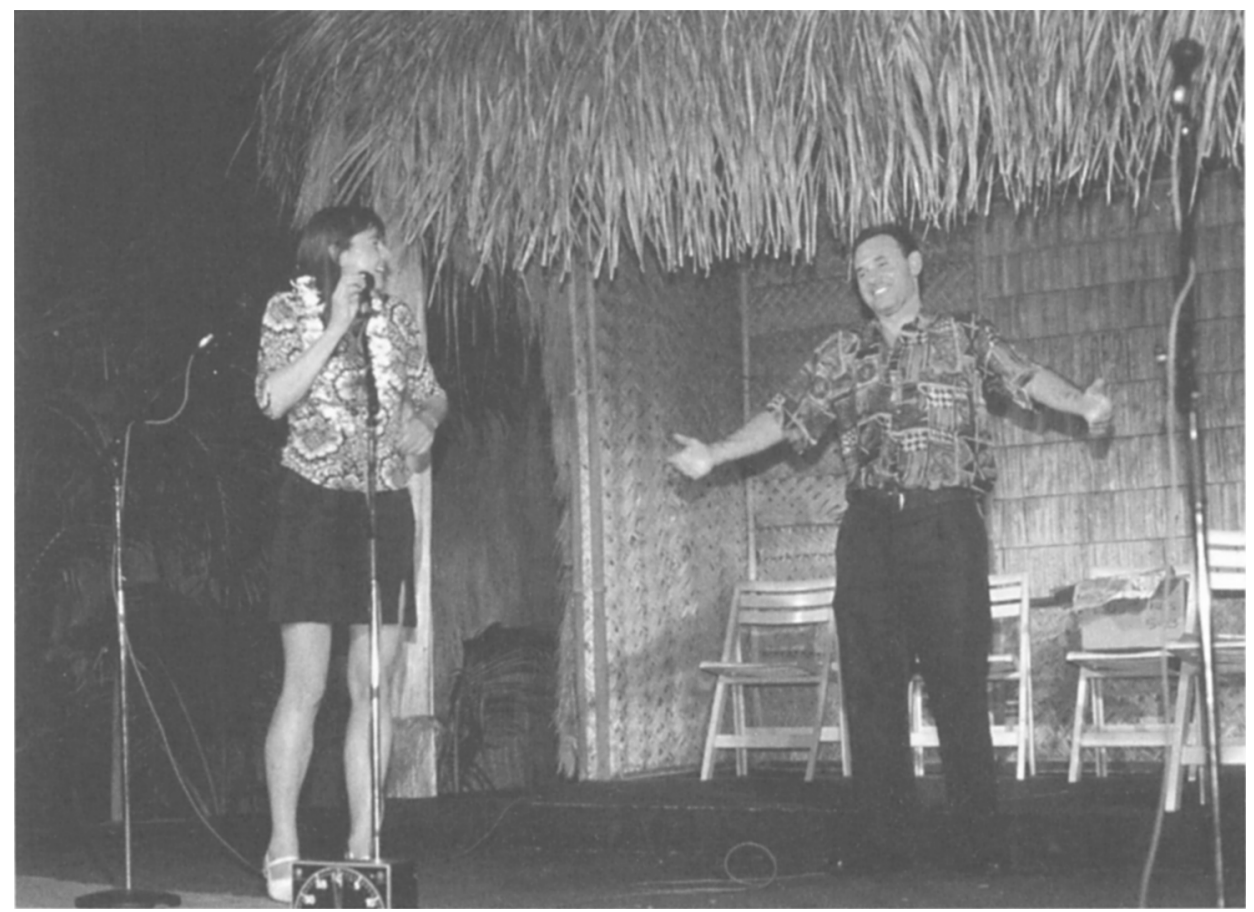

Eduardo Martín (right) strongly encourages Isabelle Baraffe to hold the next brown dwarf meeting in France. 